\title{
Technological Development Process of Emotional Intelligence as a Therapeutic Recovery Implement in Children with ADHD and ASD Comorbidity
}

\author{
https://doi.org/10.3991/ijoe.v16i03.12877 \\ Bakola N. Lizeta ${ }^{(凶)}$, Athanasios S. Drigas \\ National Center for Scientific Research "Demokritos", Agia Paraskevi, Greece \\ lizeta79@gmail.com
}

\begin{abstract}
The perception, empathy, expression and regulation of emotion have been recognized as the determining factors to everyday communication and psychosocial adaptation in children. Deficits in them can cause emotional and social problems and affect everyday life. This paper aims at investigating by reviewing the current clinical and empirical knowledge of psychoemotional and social development as much as emotional intelligence in children diagnosed with Attention Deficit Hyperactivity Disorder (ADHD) and Autism Spectrum Disorder (ASD) coexistence looking into the emotion recognition deficits as well as processing, reciprocity and emotional expression deficits noticing and characterizing these children mostly. Moreover they are considering and being studied the technical means and the ways that they could help in the development and growing of social skills and emotional intelligence. The results proclaimed that the therapeutic contribution of Information and Communication Technologies (ICTs) can be determinant.
\end{abstract}

Keywords-Emotional intelligence, artificial intelligence, emotional and social development, social interaction, ADHD and ASD comorbidity, robots

\section{Introduction}

Autism Spectrum Disorder (ASD) and Attention Deficit Hyperactivity Disorder (ADHD) are two of the most common in prevalence neurodevelopmental disorders of childhood and adulthood that can affect both instrumental and adverse impairments on cognitive and socio - emotional development [1]. Usually, these two disorders are diagnosed as separate situations, with the ASD being defined and characterized by significant impairments and deficits in mutual social interaction and communication functioning, as well as a tendency for restricted, repetitive behaviors and interests, while ADHD is characterized by inappropriate development and functionally harmful levels of hyperactivity, impulsivity, and carelessness [2]. In practice, however, these two disorders are often connected and associated.

Groom, M., et al., (2017) in their research instance that the findings of the current decade from the Autism Treatment Network database, (ATN), suggest that the coexistence of the two disorders is associated with reduced quality life and poorer 
adaptive function than any of these conditions [3]. The common significant deficits shared between the two disorders relate mainly to the emotional and social developmental part and concern difficulty in focusing and maintaining attention, in communicating with peers, in impulsivity, and varying degrees of anxiety or hyperactivity. Significant convergence and decline also occur in the perception, understanding, and expression of emotions of those who diagnosed with these kinds of disorders [4, 5]. Further neuropsychological similarities suggested in a study concerning emotional recognition and theory of mind has shown that children with ADHD were difficult to be distinguished from children with ASD [6].

The main deviances in communication and interpersonal relationships which comprise the defining characteristic of autistic spectrum disorder, (ASD), have been identified and attributed by scientists to the low levels of emotional intelligence collected by individuals of this disorder [7]. Subsequently, many researchers indicate that the social deficit which observed in children with attention-deficit and hyperactivity disorder (ADHD) has been found to be associated with their lower ability to recognize and filter others emotions resulting to promote more aggressive behavior, less tolerance in frustration as well as reduced self- regulation. The incapacity in emotional intelligence, therefore, seems to be an important correlation causal factor in the development of "abnormal" social behaviors in both disorders [8].

\section{Emotional Intelligence Contributions in Psychosocial Function of People-Emotional Intelligence Deficits in Children with ADHD and ASD comorbidity}

An individual's Emotional Intelligence (EI) is broadly defined as the ability to identify, evaluate and control the emotions of self as well as of those that interacts with. The interest and the research in EI have increased dramatically in the last decade as it responds to a variety of disorders, with the most important being the ASD and ADHD $[9,7]$.

According to Mayer \& Slovey [10], emotional intelligence is a type of social intelligence, which includes the ability of the individual to perceive and process his or her own emotions as well as those who interact with each time, in order to reach the point where he or she can be able to use the relevant information to guide his/her thinking and actions. Pursuant to their article, the field of EI is wide and contains the verbal and non-verbal evaluation and expression of emotions, the regulation of emotion for ourselves and the others and the function of emotional content in problem-solving as well. The Emotional Intelligence framework organizes existing individual bibliographic differences, regarding the ability to process and adapt an individual's personality to emotional information. Moreover, many mental problems contain emotional information that in the opinion of researchers should be processed. This procedure could be evolved completely differently in the case of the absence of non-emotional information. Mayer \& Slovey, emphasize the importance of emotional intelligence and also insist on the use of the term "intelligence" instead of "ability" with an eye to follow the terminology used in historical literature for intelligence as 
well as Gardner's example, who also gives the counterpart content of the acceptation with the term "intrapersonal intelligence" $[10,11]$.

Emotional Intelligence definition became popular by psychologist Daniel Goleman, who redefines the meaning of the term "genius" in his book which studies the importance of emotional intelligence compared to IQ [12]. In this book Goleman D., mentions five different components of emotional intelligence (Fig.1.) which are:

- Self-regulation

- Self-awareness

- Motivation

- Social skills

- Empathy.

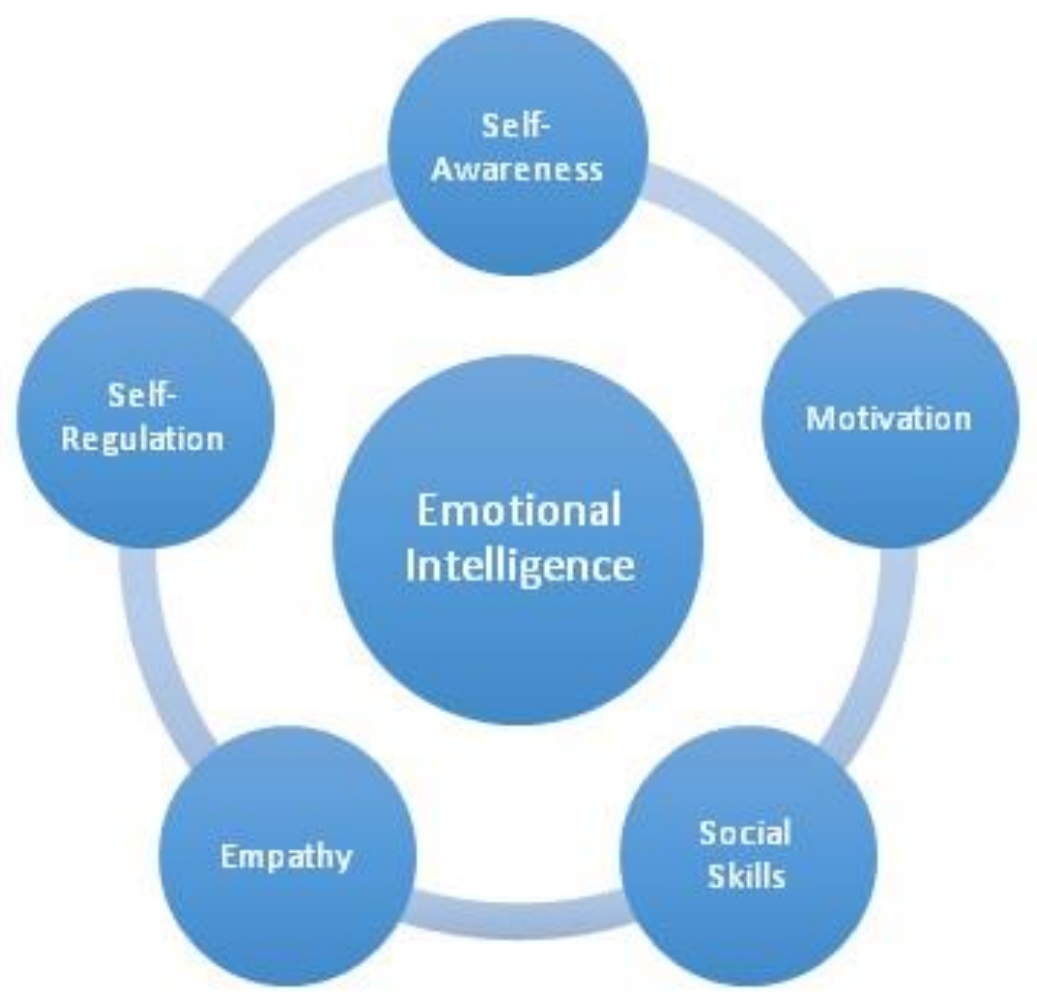

Fig. 1. The five components of Emotional Intelligence according to Goleman, D (2006) [25].

Petrides, K., V., et al., in their study mention that various aspects of emotional functioning, including accurate perception and impulsive recognition of the complex emotions of others, are diminished in people with ASD [7]. Although the ASD groups with lower functioning often used to look for social interaction and companionship, they still struggle because of their inherent difficulties in understanding the "subtle" rules of social engagement and emotions. Moreover, despite the case of a typical or 
even a high-level intelligence of people with ASD, their intact cognitive skills often allow them to develop handling strategies to obtain some "compensation" for their social difficulties, instead, the complexity of the factors that appear in social interactions as well as the demand for an immediate and rapid processing of information inevitably puts even the most capable individuals of spectrum to exhibit unstable social behavior. All these difficulties which are linked to the deficits of emotional intelligence mechanism, besides the social distress can often induce detrimental effects in people with ASD and also cause emotional conditions of anxiety and depression [7].

On the other hand, Wilkinson L., A., in his article for Emotional Intelligence (EI) in ASD, detect that social deficits that appear as its main feature are directly related to the element of emotional self- awareness which is included in the essential elements that compose the concept of emotional intelligence [9].

Emotional Intelligence deficit also seems to provoke a concern in the case of ADHD, getting at a point where scientists support that the low EI levels constitute a significant causal factor in association with non-typical social behaviors and emotional deviances both in ADHD and ASD. It is well known that children with ADHD develop interpersonal problems as also dysfunctional social behavior. These children exhibit more serious difficulties compared to their peers in empathy, less flexibility in their responses and they are less able to modify and adapt their own behaviors according to the requirements of each individual social circumstances. More generally and according to experts, these children mostly have considerable deficits in the field of self-regulation, programming and problem solving [8].

Moreover Yazdi, S. A. et al., in their research support that according to Barkley's theory, the regulation and stability of emotions require perfect executive functions but as revealed by research, children and adults with ADHD fall short of cognitive skills which demanded to control and regulate their behavior (cognitive flexibility, inhibition, monitoring, design, working memory) resulting in developing problems in emotional regulation as well. Researchers have studied the role of emotional intelligence in cognitive flexibility of children with ADHD and have concluded that deficits in both can also comprise a predictor of deficit in social, relational and ultimately in emotional and cognitive impairments in children with ADHD [13].

Finally, Waddington F., et al., indicates that the dysfunction of emotional recognition is indicated both in the heterogeneity of ASD and ASD as well as in the comorbidity type of ASD and ASD with much higher rates in the case of comorbidity. According to them, this element establishes the deficit of emotional recognition as a characteristic of ADHD and ASD [14]. The scientists emphasize the seriousness of this deficit because it can be translated into social difficulties in everyday life where a poor recording of other people's emotional expressions (either due to primary difficulties in recognizing emotions or secondary difficulties in processing information) can easily affect the social interaction of individual. 


\section{Artificial Intelligence use as a Therapeutic Intervention for Socio-Emotional Deficits in Individuals with ASD and AHD comorbidity}

The rapid rate of growth in technology-led on to the confection and creation of new technological breakthroughs that are applicable to various facets of everyday life. The use of robots for the common good nowadays has received enormous proportions and is widespread because they have the capability to serve needs in various areas of human life $[15,16]$. There are robots in almost every area of human development such as space, industry, medicine, and education. According to the scientists their abilities are excellent to such an extent that the social robots could be able to reestablish different kinds of human interactions.

The use of a Socially Assistive Robot (SAR) as a tool that could support people with ASD and ADHD to enhance their social skills or even to repair their cognitive deficits has become a purpose for many researchers [15]. The term SAR [16, 17] corresponds to the case of robots who provide assistance to their users in regulating. That means that robots have the ability to operate a variety of interactions and also can accomplish a clinical role so that the human therapists take an auxiliary position as a supporting role. However, the presence of human therapists is essential as the manipulation of robots is motivated by them.

Another similar type of SAR is that of Socially Interactive/or Intelligent Robotics (SIR). A key feature of these robots is their ability to interact with users and enhance their social skills through simulation (representation or imitation) of social interaction [16]. For effective use in the treatment of children with developmental disorders, an important feature of SAR is the variety of robots that have been purposefully designed. In particular, a wide variety of robots with different appearances have been created such as humanoid robots, animatronics robots or mechanical robots with the ability to mimic facial expressions, gestures and other physical and motor skills of humans. Both appearance and the functional elements that can be included such as lights, their distinctive voice, songs, and sounds can help to draw children's attention and provide positive reinforcement to their social interaction. In addition to their therapeutic role, they can also operate as diagnostic tools since they are able to process, measure and benchmark the deficits of users against those of individuals with typical development [17].

In recent years, many different types of social-supportive robots have been created that can be applied to the therapeutic support of children and individuals with ASD and ADHD some of which are referred to a variety of research papers and selectively are presented below.

\section{Therapeutic Robots}

Kaspar the robot who is reported by many researchers for its effectiveness in social interaction is a child-size humanoid robot designed specifically to improve the lives of children with Autism [Fig. 2. a)]. The robot has been developed by the University of 
Hertfordshire's in England world-renowned Adaptive Systems Research Group and every aspect of its appearance has been carefully studied. At first, its size is proportional to a small child for the purpose of not causing fear to its target. Afterward, it has imitative abilities and uses physical body movements and expressions but with limited facial expressions to help children to process and explicate particularly the expressions of the robot $[18,15]$. Recently Robins, B., Dautenhahn, K., \& Nadel, J., have conducted a study of 170 children and have improved that Kaspar can help children with ASD to develop communication and interactive skills by breaking isolation, encouraging the use of language, enhancing emotion recognition and by acting as a social mediator to support the best interaction between children and adults. The children display to respond much better to every environment (school, home, etc) with the presence of robot compared to the presence of a natural speaker as they appear to have fun (due to playing conditions) and eliminate the stress usually caused by situations of primary social contacts [19].

Likewise, Leonardo is a robot with progressive social skills as well as social perception, designed by the MIT Media Lab team (Massachusetts Institute of Technology) in collaboration with Stan Win Studio in order to support social interaction as well to inject empathy in children and individuals who develop deficits in these areas [Fig.2., b)], [15]. According to the MIT team, Leonardo has a variety of socio-cognitive skills and abilities. These skills and abilities include attention, mirrorneuron- inspired mechanisms to produce recognition and imitation of distinct behaviors, visual and mental abilities to be able to operate telepathically (reading human thinking) and finally some simple models of empathy.

Zeno is another advanced humanoid social robot who emerged from the collaboration between Dr. Dan Popa from the University of Texas at Arlington, with Hanson Robotics. It is a small humanoid with an expressive face designed as a human-robot interaction research platform especially for the diagnosis and treatment of ASD [Fig.2. c)]. Zeno can interact with children through non-verbal communication, with body movements and facial expressions, allowing the diagnosis of ASD even at an age in which a child is in the non-verbal stage. Milo is another robot from the same family who was created with the aim of associating and interacting directly with children [Fig.2. d)]. The main characteristic of this robot is its expressive face which has been created advisedly as children are trained to recognize the emotion that the robot exhibits each time through multiple choices on an iPad. Milo's eyes are cameras that capture the emotional feedback of the child-user with the robot. The child user wears a device on the chest that monitors the heart rate changes and therefore the feeling. Milo uses a variety of childish voices and can express or even hear a story. Both robots promote the social interaction and in particular, Milo can contribute to the development of social bonds and cultivate empathy mainly for children with ASD as well as to those who have common deficits in these areas [20, 21]. In the last five years, there has been increasing research and much new evidence on the effectiveness of Milo. Until 2017, 17 from 81 public schools of S. Carolina participated in a three-year pilot study with Milo. The research has not yet been completed but the first results already show the effectiveness of Milo [22, Fig.2. d)]. 
Following robot Nao [Fig. 2. e)] is a humanoid robot that scientists use to train children on social skills. This robot is an ideal platform for teaching and learning concepts to students of all levels. Nao can speak 19 different languages, can also walk, dance, play football, talk, listen and even recognize the people who interact with it. It is autonomous and fully programmed and is the most widely used humanoid robot in the fields of healthcare, research and education [20, 16].

Lastly, Pepper is the first humanoid social robot in the world capable of recognizing faces and basic human emotions, accepting the behavior and disposition of those who interact with [Fig. 2. f)]. Pepper is a creation of the Japanese company SoftBank and according to its creators "was designed to minimize human distress". The robot was created to provide optimized interaction with humans and is able to establish a connection with them through conversation and its touch screen. Pepper can communicate with individuals in the most natural way (typical human hand, head, and wrist movements). It has the ability to detect emotions and analyze the expressions and timbre of its interlocutor's voice and study their emotions as well. Pepper is already used in healthcare, in education, and in other positions all over the world [23, 24].

Whale A., in her article refers that in 2016 Pepper has visited a primary school in the Bristol area of the United Kingdom with the intention to interact with pupils aged 7 to 9 , as a part of a robotic course. Its effect in children, according to the class teacher, as intense as they have expressed great excitement and was constantly aiming for interacting with the robot, giving feedback to each other by asking questions to the robot or by imitating his dancing abilities and movements [24]. It is obvious that activities with social robots predispose children positively and encourage them to interact freely, limiting any inhibitions (fear, anxiety) in a safe environment.

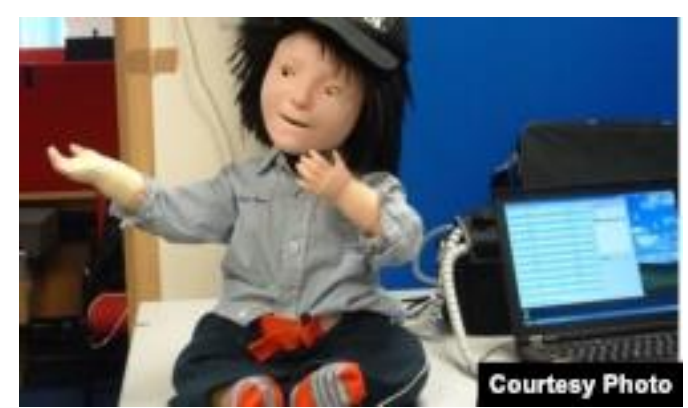

a) Kasper was made to help children with ASD [26].

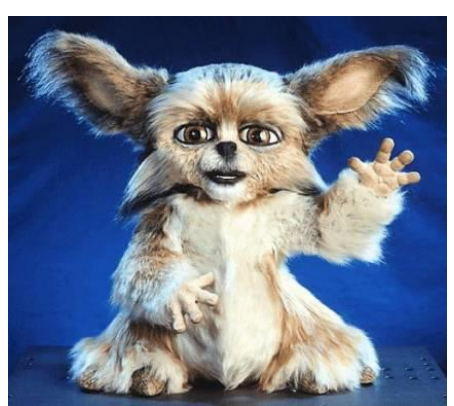

b) Leonardo, a social robot from M.I.T [27]. 


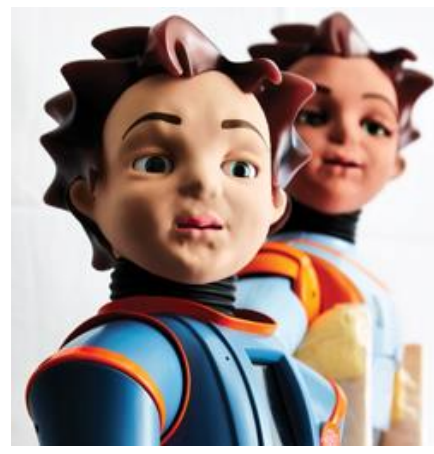

c) Zino \& Milo the humanoid robots [28].

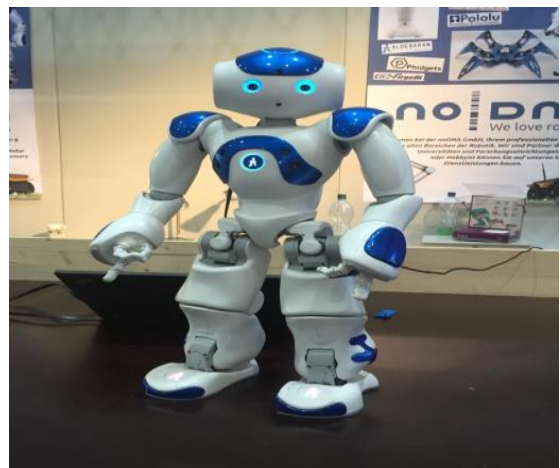

e)The robot Nao [30]

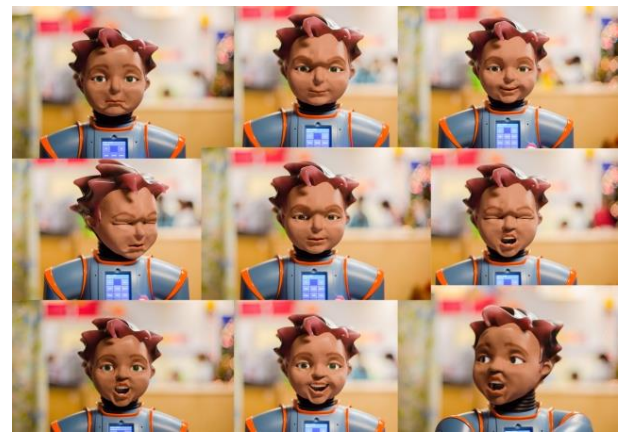

d) The many faces of Milo the Humanoid robot [29].

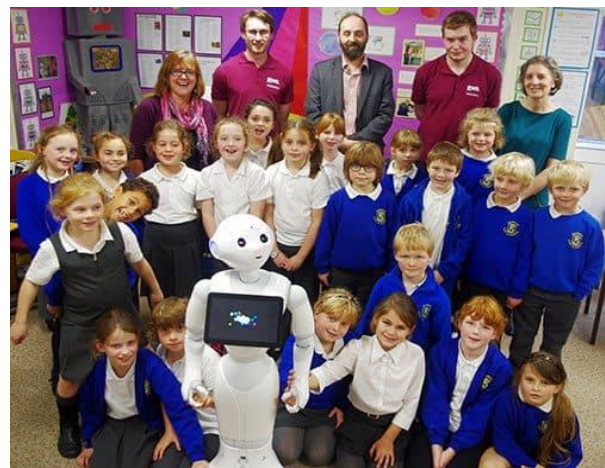

f) Pepper on the class of Bristol school children [31]

Fig. 2. Therapeutic Robots for ASD \& ADHD comorbidity

\section{Conclusion}

The main objective of this study was to review current clinical and empirical knowledge on psycho-emotional and social development as well as on the emotional intelligence of children diagnosed with the new comorbidity type of Autism Spectrum Disorder (ASD) and Attention-Deficit/ Hyperactivity Disorder (ADHD). Moreover, we tried to evaluate new technologies and more specifically the field of Artificial Intelligence (AI) as a therapeutic recovery implement for socio-emotional deficits of individuals with these disorders.

Concerning to how the emotional intelligence and the forthcoming ASD and ADHD disorders are related, it has been concluded, from the research material gathered, that the low levels of EI can commonly be found in two cases, constituting a significant causal factor in case of the development of untypical social behaviors and 
emotional deviations in both disorders while at the same time identifying deficient emotional recognition as a key future of the comorbidity type of ASD and ADHD. It is also demonstrated that the dysfunction of emotion recognition is indicated both in the heterogeneity of ASD and ADHD as in the comorbidity type of them. Finally, the severity of this deficit is also realized by the fact that it can be translated into social difficulties in everyday life as poor recording of other people's emotional expressions feels like directly affect and hold back individual's social interaction [8, 13, 7, 9, 14].

As a conclusion, on the part of Artificial Intelligence, studies have shown that social robots can function as social mediators or facilitator between a child and a teacher or a therapist by creating controlled environments that primarily aimed at reducing the stress that can be caused by typical social interactions mostly in children with ASD and ADHD. On the other hand, playing activities with social robots encourage children to interact freely, without fear, in a safe environment and reduces the tendency to social isolation promoting their sociality. Interacting with technology, in addition, eliminates the complexity and unpredictable nature of verbal or nonverbal communication that children with developmental disorders usually encounter in their interaction with other people. Considering that playing games constitute one of the most important parts of a child's cognitive and social development, the importance of technology implements is also realized [15, 17].

Finally, concerning the involvement of new technologies and their contribution to socio-emotional deficits of individuals belonging to the co-occurring type of ASDADHD, studies do not explicitly refer to the combinatorial type and therefore a further investigation is needed to ensure the most possible effective and safe intervention.

\section{$6 \quad$ References}

[1] Groom, M. J., Kochhar, P., Hamilton, A., Liddle, E. B., Simeou, M., \& Hollis, C. (2017). Atypical processing of gaze cues and faces explains comorbidity between autism spectrum disorder (ASD) and attention deficit/hyperactivity disorder (ADHD). Journal of autism and developmental disorders, 47(5), 1496-1509. https://doi.org/10.1007/s10803-017-3078-4

[2] American Psychiatric Association. (2013) Diagnostic and Statistical Manual of Mental Disorders, Fifth Edition (DSM-5), Washington, DC: American Psychiatric Association. https://doi.org/10.11

76/appi.books.9780890423349

[3] Vora P., Sikora D. (2011). Society for Developmental and Behavioral. San Antonio, TX: Pediatrics.

[4] Sivaratnam, C. S., Newman, L. K., Tonge, B. J., \& Rinehart, N. J. (2015). Attachment and emotion processing in children with autism spectrum disorders: Neurobiological, neuroendocrine, and neurocognitive considerations. Review Journal of Autism and Developmental Disorders, 2(2), 222-242. https://doi.org/10.1007/s40489-015-0048-7

[5] Davis, N. O., \& Kollins, S. H. (2012). Treatment for co-occurring attention deficit/hyperactivity disorder and autism spectrum disorder. Neurotherapeutics, 9(3), 518530.https://doi.org/10.1007/s13311-012-0126-9

[6] Buitelaar, J. K., Van der Wees, M., Swaab-Barneveld, H. A. N. N. A., \& Van der Gaag, R. J. (1999). Theory of mind and emotion-recognition functioning in autistic spectrum 
disorders and in psychiatric control and normal children. Development and psychopathology, 11(1),39-58. https://doi.org/10.1017/s0954579499001947

[7] Petrides, K. V., Hudry, C., Michalaria, G., Swami, V., \& Sevdalis, N. (2011). Comparison of the trait emotional intelligence profiles of individuals with and without Asperger syndrome. Autism, 15, 671-682. https://doi.org/10.1177/1362361310397217.

[8] Kaypakl1, G. Y., \& Tamam, L. (2019). Emotional Intelligence in Attention Deficit Hyperactivity Disorder. Current Approaches in Psychiatry/Psikiyatride Guncel Yaklasimlar, 11(1).https://dergipark.org.tr/tr/download/article-file/612291. https://doi.org/10.5455/cap.20130726020101

[9] Wilkinson, L., A., (2013).Emotional Intelligence (EI) and ASD. Living Autism. http: //livingautism.com

[10] Mayer, J. D., \& Salovey, P. (1993). The intelligence of emotional intelligence.

[11] Gardner, H. (1983). Frames of mind. New York: Basic Books.

[12] Goleman, D. (2006). Emotional intelligence. Bantam.

[13] Yazdi, S. A. A., Farahi, S. M. M., Farahi, S. M. M. M., \& Hosseini, J. (2018). Emotional Intelligence and its role in Cognitive Flexibility of Children with and without Attention Deficit Hyperactivity Disorder. Anales De Psicología/Annals of Psychology, 34(2), 299304.http://scielo.isciii.es/pdf/ap/v34n2/0212-9728-ap-34-02-298.pdf. https://doi.org/10.6018/analesps.34.2.283771

[14] Waddington, F., Hartman, C., de Bruijn, Y., Lappenschaar, M., Oerlemans, A., Buitelaar, J., \& Rommelse, N. (2018). An emotion recognition subtyping approach to studying the heterogeneity and comorbidity of autism spectrum disorders and attentiondeficit/hyperactivity disorder. Journal of neurodevelopmental disorders, 10(1), 31. https://doi.org/10.1186/s11689-018-9249-6

[15] Vélez, P., \& Ferreiro, A. (2014). Social robotic in therapies to improve children's attentional capacities. Review of the Air Force Academy, (2), 101.

[16] Cho, S. J., \& Ahn, D. H. (2016). Socially assistive robotics in autism spectrum disorder. Hanyang Medical Reviews, 36(1), 17-26. https://doi.org/10.75 99/hmr.2016.36.1.17

[17] Fridin, M., \& Yaakobi, Y. (2011). Educational Robot for Children with ADHD/ADD, Architecture Design.

[18] Miller, B., 2018. How Kaspar the robot is helping autistic students to socialize. ABC.net.https://www.abc.net.au/news/2018-06-05/the-creepy-looking-robot-teachingkids-social-skills/9832530.

[19] Robins, B., Dautenhahn, K., \& Nadel, J. (2018). Kaspar, the social robot and ways it may help children with autism-an overview. Enfance, (1), 91-102. https://doi.org/10.3 917/enf2.181.0091

[20] Yousif, J. H., Kazem, H. A., Chaichan, M.T., (2019) Evaluation Implementation Of Humanoid Robot For Autistic Children: A Review. International Journal of Computation and Applied Sciences 6(1):412-420

[21] Tucker, E., (2015). How robots are helping children with autism. The Guardian, international edition. https://www.theguardian.com/lifeandstyle/2015/feb/01/how-robotshelping-children-with-autism.

[22] Mills, B., (2017). Meeting Milo: Robot uses spectrum of facial expressions to engage with autistic students at Kingsbury Elementary. The Sumter ITEM. https://www.theitem.com/stories/meeting-milo-robot-uses-spectrum-of-facial-expressionsto-engage-with-autistic-students-at,300398?platform=hootsuite.

[23] Malewar Amit. (2019). Meet Pepper: The Friendly Humanoid Robot. Robotics. Tech Explorist. 
[24] Whale Alice., (2016). Updated: Bristolrobotics firm inspires local school children with humanoid robot. Tech Spark. .

[25] http://theimportanceofemotionalintelligence.weebly.com/the-5-components.html

[26] https://learningenglish.voanews.com/a/health-lifestyle-robot-helps-autistic-children-learnsocial-skills/3801379.html

[27] https://www.researchgate.net/publication/284185809_Social_Learning_for_Persinalizaatio n_in_HRI/figures?lo=1

[28] http://www.roboticare.nl/zeno-milo/

[29] https://www.theitem.com/stories/meeting-milo-robot-uses-spectrum-of-facial-expressionsto-engage-with-autistic-students-at,300398

[30] https://en.wikipedia.org/wiki/Nao (robot)

[31] https://www.techspark.co/blog/2016/11/22/bristol-robotics-firm-inspires-local-schoolchildren-humanoid-robot

\section{$7 \quad$ Authors}

L. Bakola has a M.Sc. degree in "Expertise in ICTs and Special Education Psychopedagogyof Integration" from the Democritus University of Thrace and she is a research associate at N.C.S.R. "Demokritos", IIT- Net Media Lab \& Mind - Brain R\&D, Agia Paraskevi, 153 10, Athens, Greece (lizeta79@gmail.com).

A. Drigas is a Research Director at N.C.S.R. "Demokritos", IIT- Net Media Lab \& Mind - Brain R\&D, Agia Paraskevi, 153 10, Athens, Greece (dr@iit.demokritos.gr).

Article submitted 2019-12-24. Resubmitted 2020-01-14. Final acceptance 2020-01-15. Final version published as submitted by the authors. 